\title{
Lectura crítica de medios en textos escolares desde la comunicación educativa ${ }^{1}$
}

\author{
Recibido: 08 de septiembre de 2014 \\ Aceptado: 29 de mayo de 2015 \\ Publicado: 30 de octubre de 2015
}

\author{
Ximena Ojeda Sánchez \\ ximenaojedasanchez@gmail.com \\ Carlos del Valle Rojas \\ carlos.delvalle@ufrontera.cl. \\ Juan del Valle Rojas \\ dvalle1985@gmail.com \\ Universidad de La Frontera (Chile)
}

Resumen: Este artículo presenta los resultados de la investigación acerca del concepto de lectura crítica de medios de comunicación en textos escolares de séptimo básico a segundo medio, a través de una mirada desde la comunicación y la educación. El objetivo es conocer el concepto de lectura crítica de medios que subyace en los textos escolares entregados por el Ministerio de Educación para, además, establecer los niveles de alfabetización mediática que proponen los contenidos y actividades insertos en estos recursos de aprendizaje. Los resultados nos permiten señalar que la metodología propuesta para la enseñanza de lectura crítica de medios en textos escolares apunta a una educación "a través" de los medios, predominando el nivel funcional instrumental de los medios en todos los textos escolares analizados.

Palabras clave: Lectura crítica de medios, alfabetización mediática, textos escolares.

\begin{abstract}
This article focuses on the results of a research on the concept of critical reading of the media in school coursebooks from seventh grade in primary school to second grade in secondary school, by considering the point of view of the communication and educational areas. The aim is to know the concept of critical reading that is present in the coursebooks
\end{abstract}

1 Este artículo forma parte del proyecto FONDECYT N ${ }^{\circ} 1110711$. 
given by the Ministry of Education, in order to establish the levels of media alphabetization that are given by the several contents and activities inside the learning resources. The results indicates that the methodology proposed for the teaching of the media's critical readings in coursebooks focuses on an education "through" the media, by emphasizing the functional and instrumental level of the media in all of the coursebooks that were analyzed.

Key words: Critical Lecture of the Media, Media Alphabetization, Coursebooks.

\section{Introducción}

La Reforma de Educación de 1990 incluyó dentro de los ejes de enseñanza del subsector de Lenguaje y Comunicación, a los medios masivos de comunicación, insertos en el Decreto de Educación 22, del año 1998, en el que se establecen los objetivos fundamentales y contenidos mínimos obligatorios y se fijan normas generales para su aplicación. Sin embargo, con el ajuste curricular del 2009, se realizan modificaciones en el subsector de Lenguaje y Comunicación en torno a tres ejes: Comunicación Oral, Lectura y Escritura, con lo cual se elimina el eje de medios masivos de comunicación: "Lo importante es dejar en claro que los medios de comunicación también están al servicio de las necesidades formativas del sector y propician actividades de lectura, escritura y comunicación oral en torno a ellos" (MINEDUC, 2009: 34).

Por su parte, los textos escolares analizados corresponden a aquellos entregados por el Ministerio de Educación al año 2012, fecha de inicio de la investigación, correspondiente a las editoriales MN para $7^{\circ}$ básico y Santillana para los demás niveles. La selección del corpus responde a los cursos en los cuales se están aplicando los ajustes curriculares; en tanto, el análisis de datos se realiza en base a la matriz de recogida de datos de los niveles de alfabetización mediática y competencia mediática, elaborada para cumplir con los objetivos de este proyecto.

\section{Marco teórico}

\subsection{Lectura crítica de medios como marco operacional}

Antes de abordar el concepto de lectura crítica de medios, es pertinente desglosar el concepto para aproximarnos a las nociones que lo componen. Para Serrano de Moreno y Madrid de Forero (2008: 61), la lectura crítica favorece la formación de "ciudadanos capaces de interpretar los valores dominantes, identificar puntos de vista y desentrañar intencionalidades, imaginarios e ideologías que subyacen en la diversidad de textos que circulan en la vida social".

Este concepto no es restrictivo, en lo absoluto, porque abarca todos los tipos de textos, de distintas intencionalidades, géneros, formatos, entre otros, lo que amplía la concepción tradicional de lectura, es decir, hay que considerar que si hablamos de lectura crítica, 
necesariamente hemos de considerar la idea de lectura o, más bien, de lecturas posibles. Del mismo modo, cuando hablamos de medios masivos de comunicación, han de considerarse en su complejidad y multimodalidad.

Ahora bien, la lectura crítica constituye una dimensión de la alfabetización crítica (Cassany, 2004; Fainholc, 2005; Luke, 1997), pero con lo anterior surge la pregunta: ¿Qué entenderemos por crítica, lo crítico o criticidad? No es un asunto menor, considerando que además de definir qué es la crítica, se requiere reflexionar acerca de cómo se vincula esta noción con la alfabetización crítica, en el contexto educacional. Pues bien, implica reflexionar acerca de lo que se presenta, sin aceptarlo como "verdad absoluta", sino más bien como una condición de posibilidad, un "mundo posible"; de tal manera que podamos develar intencionalidades y relacionarlas con las visiones personales, para así adoptar posturas más conscientes. Para Cassany (2006: 48), es central destacar el valor de la lectura para la vida en democracia: "La democracia se basa también en la capacidad de comprender, en habilidades de lectura, comprensión y reflexión de los ciudadanos".

Pensando en el contexto educativo, ¿por qué considerar la lectura crítica como una competencia formativa? Porque la lectura crítica se enmarca dentro de las competencias cognitivas y pragmático-culturales, esto último en tanto que "son aquellas que muestran la capacidad del lector para identificar los propósitos del discurso, sus usos y funciones, sus orígenes, de acuerdo con el contexto sociocultural e ideológico en que fue creado y formular propuestas o hacer uso de las ideas y representaciones en variados entornos culturales y sociales" (Serrano de Moreno y Madrid de Forero, 2008: 65).

Esto implica reflexionar acerca de las ideologías que subyacen en los discursos, representaciones sociales, entre otros, pero no de manera aislada, sino considerando el contexto de producción y recepción. Es decir, como competencia posee una finalidad funcional para los estudiantes, centrándose también en la praxis. Por otra parte, Sánchez y Aguaded (2009: 138) realizan un proceso de aprendizaje sobre la televisión, destinado a niños entre siete y once años para el cual definen una de las variables (la lectura crítica) precisando:

- "Definición conceptual: es la capacidad de descubrir la finalidad y la estructura de un documento audiovisual, llegando incluso a proponer cambios en dicho espacio.

- "Definición operacional: Se podrá afirmar que un niño ha realizado una lectura crítica elemental de un documento audiovisual, si reconoce aspectos temáticos, técnicos y propone cambios en su estructura, según corresponda al género visualizado".

Si bien esta propuesta se aplica a la televisión, es pertinente como marco conceptual para esta investigación. Lo más rescatable se plantea a nivel de cambios posibles de manifestar por parte de los niños, es decir, la capacidad de transformar. Se trata "no de descubrir las facetas tecnológicas del medio [...] sino más bien de revelar los aspectos del medio que 
repercuten en una mejora de la competencia, en su doble dimensión de lectura crítica y escritura creativa" (Aguaded, 1997). Lo anterior, entonces, se relaciona con la concepción de la educación "sobre" y "en" los medios, planteando una interacción que implique, por un lado, su análisis y consiguiente reflexión; y por otro, un uso creativo de estos, de manera de apropiarse de ellos y lograr ciertos niveles de transformación social.

Considerando que proponemos la lectura crítica de medios como competencia, la ubicamos dentro de la lectura crítica, como competencia genérica, que no se restringe sólo al análisis y comprensión de los medios masivos de comunicación. La lectura crítica de medios es una competencia para comprender la dimensión de contenidos, los que operan en dos niveles:

- Discursivo y de sentido: los discursos reflejan el orden social establecido, las relaciones de dominación, las injusticias y las opresiones. A partir de este axioma, comprender críticamente significa identificar este conjunto de presupuestos y poder confrontarlo con otras alternativas (Cassany, 2006: 86). Es decir, parten desde la reflexión y análisis de los distintos lenguajes utilizados por los medios de comunicación para (desde ahí) develar e inferir lo dicho y lo no dicho.

- Material y de poder: tal es el impacto que han tenido los medios, que se afirma que la racionalidad de las relaciones sociales se construye hoy en base a la lucha por dominar los medios de distinción simbólica sobre los medios de producción material (García-Canclini, 1995). De acuerdo con la concepción de Pérez Tornero (1994: 28), entendemos por lectura crítica, en definitiva, "aquella que busca aprovechar la propuesta de sentido de la televisión como una oportunidad para la recreación, para la reinterpretación, para el juego inteligente del sentido. En unos casos, esto se resolverá contra las propuestas del propio mensaje. Pero en otros, caminará en su misma orientación y aprovechará lo que de positivo hay en él. La lectura crítica es aquella que lucha contra la univocidad y el monolitismo en la interpretación. La que no acepta la pretendida transparencia de su mensaje y se cuestiona, en cambio, la influencia en él de la mediación, es decir, del punto de vista del emisor". En síntesis, el emisor también constituye un mediador entre los mensajes de los medios y la realidad.

\subsection{La alfabetización mediática como marco de referencia}

La lectura crítica de medios como competencia solo puede estudiarse en términos de la actuación, es decir, a través de procedimientos y aprendizajes concretos: "Todos estos principios de actuación -chomskianos y habermasianos- se reducen en uno: potenciar una nueva competencia comunicativa" (Pérez Tornero, 1994: 34).

La noción de alfabetización mediática no ha sido consensuada aún, ya que es un tema cuya amplitud está en discusión, sus límites y las relaciones con otros conceptos afines, como educación para los medios y lectura crítica de medios. De hecho, se habla de "alfabetización 
audiovisual" o "alfabetización mediática", según distintos autores (Buckingham, 2005; Kellner y Share, 2007; Livingstone, 1999; 2004; 2007; Zarandona y otros, 2008). Sin perjuicio de ello, el concepto busca comprender el proceso mediante el cual se dota a los individuos, por ejemplo en el sistema educacional formal, de los "conocimientos, las habilidades, y las competencias que se requieren para utilizar e interpretar los medios" (Buckingham, 2005: 71); y para ello es preciso realizar una incorporación contextualizada (Del Valle, 2005).

El concepto de alfabetización ha sufrido variaciones en el transcurso del tiempo; especialmente debido al contexto actual, caracterizado por profundos cambios sociales y culturales provocados por la globalización y el desarrollo de las TIC; no obstante, continúa manteniendo un aspecto transversal, ya que para Bazalgette, "la alfabetización mediática se ha enmarcado en la tendencia propia del siglo XX de añadir el término 'alfabetización' con el fin de impulsar ciertas áreas como aspectos esenciales del aprendizaje" (ápud Murphy, 2010: 24).

Ahora bien, se concibe la alfabetización como un proceso (similar al de educar), más que como un "resultado". Alfabetizar implica enseñar ciertos contenidos, procedimientos, pero no apostamos porque una persona alfabetizada está necesariamente en condiciones de realizar lectura crítica de medios. Lo anterior indica que el término "alfabetización" es dinámico y cambiante, de acuerdo con las competencias básicas (mínimas) que deben ser adquiridas en distintos escenarios socioculturales.

\subsection{1. ¿Qué es la alfabetización mediática?}

Entenderemos la alfabetización mediática como el conjunto de conocimientos, habilidades, y competencias necesarias para usar e interpretar los medios de comunicación masivos. Puede subdividirse en niveles de desempeño, en tanto la alfabetización mediática se concibe como proceso.

\subsubsection{Niveles de desempeño de la alfabetización mediática}

- Nivel funcional: implica un nivel básico de alfabetización mediática. Específicamente, se relaciona con una comprensión de los medios masivos basada exclusivamente en su funcionamiento, es decir, como herramientas para la producción y difusión.

- Nivel analítico-descriptivo: refiere a los lenguajes y tecnologías en tanto procesos de producción y recepción.

- Nivel crítico-comprensivo (de las mediaciones): implica un nivel avanzado de alfabetización mediática. En este nivel se aprecia una reflexión profunda sobre los medios y su influencia, tanto en el entorno como en uno mismo, y una búsqueda activa por encontrar mecanismos que minimicen el 
impacto negativo de los medios, aprovechando al máximo su lado positivo. Permite reconocer el poder que ejercemos sobre los medios, y fortalecer la participación activa y responsable en la fiscalización de los medios como otra expresión de ciudadanía.

\section{Metodología}

\subsection{Técnica preliminar: Método Delphi}

Para establecer una aproximación inicial a la noción de lectura crítica de medios, se aplicó el método Delphi como proceso sistemático y grupal "encaminado hacia la obtención de las opiniones y del consenso, a partir de las experiencias y juicios subjetivos de un grupo de expertos" (Pozo, Gutiérrez y Rodríguez, 2007: 353).

Por lo anterior, este se presenta como un método idóneo para acercarnos al objeto de estudio no solo desde un lente teórico, sino desde las miradas de otros actores vinculados a la educación en medios. Así, se consideraron las opiniones recogidas de dos representantes de cada una de estas áreas:

- Investigadores de comunicación-educación, a nivel nacional e internacional: investigadores de la especialidad en el primer y segundo caso.

- Profesores sin estudios de posgrado que ejercen la labor docente en enseñanza media, con al menos 5 años de experiencia: profesores de la especialidad.

- Estudiantes egresados de cuatro medio que aún no ingresan a estudios superiores al año 2012.

Esta técnica se aplicó a través del envío por correo electrónico de una pregunta global a cada una de las personas para obtener la información requerida. Esta fue la misma para investigadores y profesores, pero fue reformulada para estudiantes egresados de enseñanza media para asegurar su cabal comprensión.

- Para estudiantes

Pregunta: "Considerando el contexto educativo nacional, ¿qué crees tú que es la lectura crítica de medios?, ¿qué contenidos debiese considerar y cómo debiese ser enseñado en los textos escolares?".

- Para investigadores y profesores

Pregunta: "Considerando el contexto educativo nacional, tanto en lo político general como en lo específico en aula, ¿cuál es la noción de 'lectura crítica de medios' a aplicar como contenido y didáctica en los libros de texto de primero a cuatro medio?". 
Tomando la respuesta de cada persona, se sistematizó la información en una tabla como la que se presenta:

Figura 1: Mapa contextual para el desarrollo del periodismo de datos.

\begin{tabular}{|l|l|l|l|}
\hline & Definición & $\begin{array}{l}\text { Contenidos } \\
\text { vinculados }\end{array}$ & Didáctica \\
\hline & & & \\
\hline
\end{tabular}

Fuente: Elaboración propia.

\subsection{Técnicas de procesamiento de la información: análisis de los datos}

El análisis de contenido es una técnica empleada en investigación cualitativa para transformar los datos de campo recogidos en información y conocimiento. La realidad proporciona datos a partir de los cuales el investigador elabora el conocimiento que explica o comprende la realidad misma. Sin datos, no hay conocimiento científico. En efecto, los datos no tienen valor científico por sí mismo, puesto que son propiedades o características que pertenecen a los hechos, están en ellos, de forma tal que es el sujeto o investigador quien los recoge (abstrae), les asigna valor o significado y los convierte en información cognitiva.

En este sentido, esta técnica resulta la más apropiada a la hora de profundizar en los datos que se extraen de la experiencia de cada estudiante, ya que nos permite como investigadores interpretar la realidad de acuerdo a las circunstancias y particularidades de su contexto. Para Bardin (2002), el análisis de contenido cualitativo permite ver la presencia o ausencia de una característica o de un conjunto de características de contenido dadas en un cierto fragmento de mensaje que es tomado y considerado para el estudio.

Como técnica específica de levantamiento de datos, se ha utilizado una matriz de recogida de datos de los niveles de alfabetización y competencia mediática, la cual ha sido elaborada por Del Valle, basado en los textos de Giménez (1981) y Bonilla (2006), a fin de cumplir los propósitos de esta investigación y se encuentra compuesta por los siguientes puntos:

- Referencia al texto: datos formales del libro, tales como autor, año, editorial.

- Contenidos/actividades encontradas: referencia a todos los contenidos y actividades relacionados con los medios masivos de comunicación que aparecen en el libro. Incluye lista de unidades, subunidades, tópicos, ejercicios, etc. A su vez, se etiquetan por contenido (C) o actividad (A), según corresponda.

- Descripción de los contenidos/actividades: Breve descripción de lo que se señala explícitamente en cada contenido y actividades ya mencionadas (citas textuales entrecomilladas). La referencia debe indicar qué se dice, de qué o de quién. 
Solo en algunas ocasiones no se establece cita textual, en los casos que no haya información que sea representativa. Se consigna con:

- Nivel funcional-instrumental (de los medios): por ejemplo, referencias a los lenguajes y tecnologías, en tanto herramientas para la producción y difusión.

- Nivel analítico-descriptivo (de los medios): por ejemplo, referencias a los lenguajes y tecnologías, en tanto procesos de producción, recepción e interacción.

- Nivel crítico-comprensivo (de las mediaciones): por ejemplo, referencias a los procesos de producción, recepción e interacción, incluyendo aspectos ideológicos, valóricos y estéticos.

\subsection{Técnica de análisis de datos}

Tomando los datos recogidos en la matriz, estos se cuantifican por contenido (C) y actividad (A), lo que da como resultado un listado de tipologías textuales, que se han consignado con su respectiva nomenclatura, a saber:

- Texto digital, blog, Power Point, Word y otros similares (TD).

- Carta al director (CD).

- Texto informativo, noticia (TI).

- Reportaje (RE).

- Entrevista (EN).

- Afiche, publicidad con contenido con fines de lucro y publicidad sin fines de lucro (CL, SF).

- Comentario crítico (CC).

- Radioteatro (RA).

- Editorial (ED).

- Ficha técnica: se refiere al formato de ficha para presentar algún contenido, como el caso de fichas de animé y elaboración de la misma solo en el caso que contenga información referida a medios masivos de comunicación $(\mathrm{F})$.

Otras tipologías textuales presentan la siguiente subdivisión:

- Reflexivas: corresponden a actividades como preguntas introductorias (actividad inicial) o textos que invitan a reflexionar sobre situaciones cotidianas referidas a temáticas asociadas a los medios de comunicación, consumo de medios y otros. Se consigna con la letra (R).

- Hipervínculo: el concepto de hipervínculo proviene de la web, pero autores como Gilster (1997) atribuyen sus inicios al texto escrito, con el uso de notas a pie de página. En palabras de Pujolà y Montmany (2010: 3) son aquellos enlaces que "permiten enlazar información de manera no secuencial dejando al lector la opción de decidir su lectura y su relación con el texto". Por lo anterior, el hipervínculo 
es entendido como lógica de organización del texto multimodal ${ }^{2}$ y no como tipo textual. Se refiere a las estrategias pedagógicas utilizadas en los textos escolares, de las cuales se traspasa, principalmente a actividades de Internet. Por ejemplo, los apartados denominados "Para investigar", en los que se invita a profundizar sobre distintos temas mediante la búsqueda en Internet; o "Para ver", en el que se sugieren películas para que los estudiantes complementen sus aprendizajes. Se consigna con la letra $(\mathrm{H})$.

Cada una de estas tipologías ha considerado la subcategoría de (C) y (A). Cabe destacar que al no constituir tipos textuales específicos, pueden integrar textos de otra categoría, como en el caso de preguntas acerca del uso de herramientas en Internet. Una vez que establecen estas tipologías textuales, se cuantifica la recurrencia de cada una en los cuatro textos escolares analizados en esta investigación, los que se sistematizan en la tabla que se presenta en los resultados. Posteriormente, se seleccionan los textos más recurrentes: texto digital, entrevista y afiche (publicidad con fines de lucro y publicidad sin fines de lucro) y se realiza un análisis de su proyección en los cursos que atañen a esta investigación.

\section{Resultados: análisis de tipologías textuales recurrentes por nivel}

Los resultados obtenidos a partir de la investigación y el análisis cualitativo del corpus se presentan considerando las tipologías textuales emergentes con mayor aparición y frecuencia. Posteriormente, se presentan gráficos de los niveles de alfabetización mediática. Para efectos de esta investigación, se realiza un análisis descriptivo de la presencia de los tipos de texto y de su proyección en los cursos que atañen a esta investigación, solo en los casos más recurrentes en los cuatro niveles, de manera de establecer resultados significativos.

\subsection{Texto digital}

El texto digital ha sido el predominante en el análisis de los datos recogidos. Como se aprecia en el gráfico, tanto los contenidos como las actividades asociadas presentan un incremento progresivo y constante en el transcurso de los cuatro niveles, llegando a las 48 actividades en $2^{\circ}$ medio.

2. Al analizar los textos escolares hay que comprender que éste es multimodal, siguiendo a Pujolà y Montmany (2010), "entendiendo texto en su sentido más amplio, no sólo el texto lingüístico". 
Figura 2: Texto digital (por contenido y actividad).

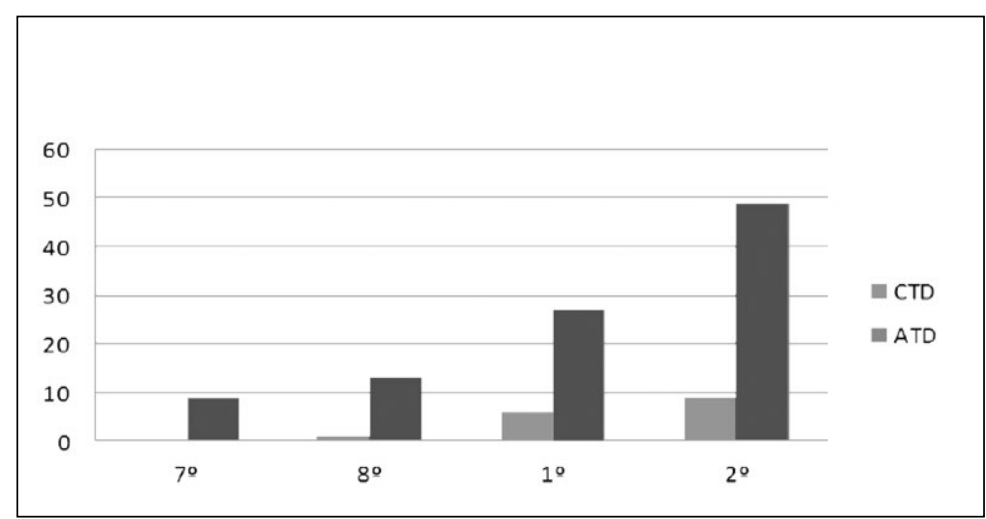

Fuente: Elaboración propia.

En relación a la metodología, en $7^{\circ}$ básico no se imparten contenidos, pero estos se presentan en mayor cantidad cada año, hasta finalizar en $2^{\circ}$ medio con 9 de ellos. Es decir, existe una estrategia paulatina en el aumento de contenidos, según el desarrollo cognitivo de los estudiantes. Dentro de lo que atañe al texto digital, predominan actividades consignadas como "transcripción a texto digital" del tipo: "utiliza los recursos de autotexto que provee el procesador de textos y los recursos de alineación de párrafo" o en la "barra de herramientas" tienes la opción "dibujo" y en ella "autoformas". Acá puedes usar las "llamadas" y "cintas y estrellas" para crear tus "globos de texto" ( $c f$. Watson y Aguilera, 2012). Es decir, se centra en el nivel funcional-instrumental de los medios, lo que ha sido consignado como "manejo de formatos digitales de escritura".

\subsection{Entrevistas}

En el caso de la entrevista, se omiten contenidos en $7^{\circ}$ básico, pero estos aumentan en $8^{\circ}$ y $1^{\circ}$ medio con un contenido en cada caso. No obstante, las actividades se presentan desde $7^{\circ}$ a $1^{\circ}$ medio, pero el incremento no es gradual. La mayor cantidad se concentra en $1^{\circ}$ medio (10) y en $7^{\circ}$ básico (5); en tanto, $8^{\circ}$ básico presenta 4 actividades.

La entrevista no se trabaja en $2^{\circ}$ medio, ni como contenido ni como actividad. Para esta tipología, se proponen actividades del tipo: "Haz de cuenta que vas a entrevistar a un personaje destacado" (ibídem) y "¿Cuál de las siguientes opciones expresa mejor el tema principal del texto?” (Vidal y González, 2011). 
Figura 3: Entrevista (por contenido y actividad).

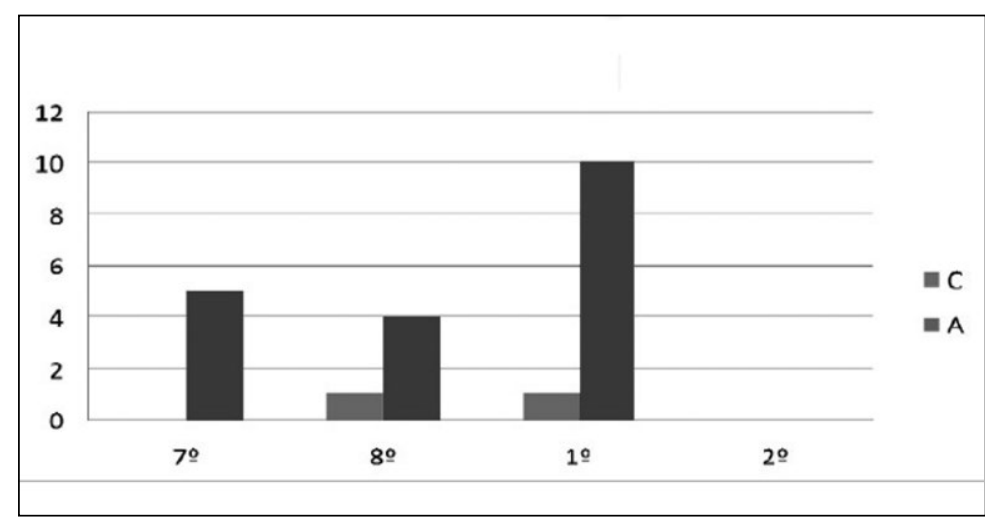

Fuente: Elaboración propia.

\subsection{Afiche: publicidad con fines de lucro}

En el caso denominado "publicidad" en los textos escolares, se percibe la predominancia de contenidos, los cuales se presentan en $8^{\circ}$ básico, $1^{\circ}$ y $2^{\circ}$ medio. Las actividades, en cambio solo se presentan en $1^{\circ}$ medio (3), presentándose omisión en los demás niveles. Se destaca el caso de $7^{\circ}$ básico, en el cual no hay contenido ni actividades. Lo anterior encuentra su causa en el año 2011, ya que los textos escolares y su confiabilidad estuvieron en el centro de la polémica, porque se denunció que éstos contaban con publicidad de marcas nacionales, canciones y slogan. Ante esto: "El ministerio de Educación prohibió el uso de publicidad en los textos escolares y la aparición de marcas luego que esta jornada se conociera que libros para menores de 12 años presentaban tal condición" (El Mercurio, 5/ IV/2011). Los cambios se aplicaron desde el año 2012.

Figura 4: Afiche: publicidad con fines de lucro (por contenido y actividad).

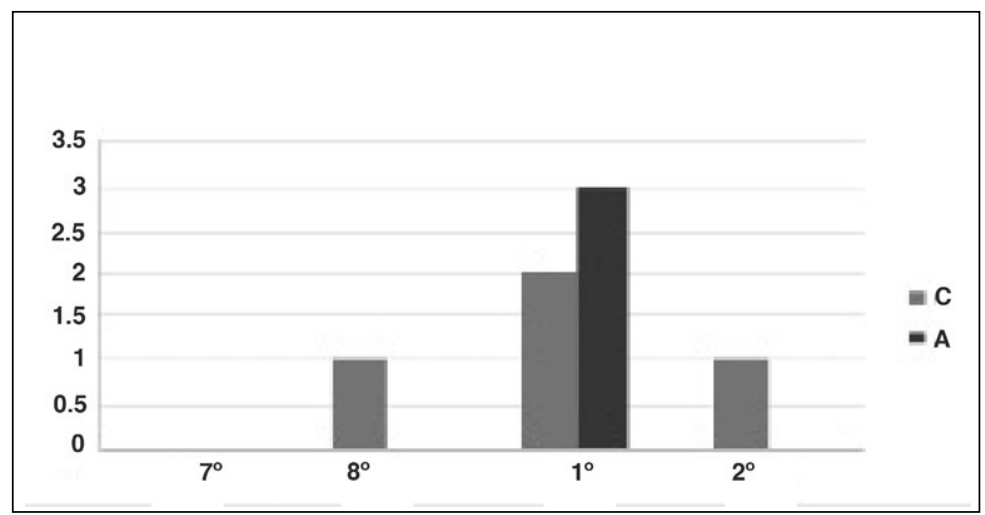

Fuente: Elaboración propia. 
"En adelante, los textos que hagan referencias a contenidos de publicidad, y que vienen editándose desde años anteriores, no pueden hacer alusión a marcas específicas de productos" (ibídem). Esta instrucción estaba contenida en el oficio a cargo del entonces Ministro de Educación, Joaquín Lavín. Por ello, los textos ya no cuentan con publicidad de marcas específicas. Sin embargo, este extremo de utilizar el mecanismo discursivo de omisión no contribuye al desarrollo de habilidades de lectura crítica; en un tipo de texto habitual y que cuenta con la causa que explica por qué se omiten o eliminan estos contenidos. Así, no debía eliminarse, en tanto es "una de las formas más exquisitas de encubrimiento pero a la vez, recubrimiento del poder real" (ibídem), aunque en sus inicios, publicitar se basó en el ejercicio evidente de promoción de bienes materiales y/o simbólicos, pero luego, comienzan las sutilezas comunicativas que hacen más compleja la comprensión y lectura crítica de medios. Pineda Cachero (2012: 196) radicaría en que "los ideales de una educación que fomente el libre desarrollo del educando se enfrentan inevitablemente con las constricciones de las necesidades del poder" y la educación ha sido la gran herramienta de control del conocimiento y del ocultamiento del mismo.

\subsection{Afiche: publicidad sin fines de lucro}

La publicidad sin fines de lucro es lo que en los textos escolares se denomina "propaganda" y que se define como aquella que intenta influir en la ideología de los receptores. Acerca de los contenidos y actividades de los afiches como tipología textual (específicamente en los que constituyen publicidad con contenidos sin fines de lucro) se percibe omisión de estos; lo que coincide con los contenidos ni actividades asociados a la temática de publicidad sin fines de lucro.

Figura 5: Afiche: publicidad sin fines de lucro (por contenido y actividad).

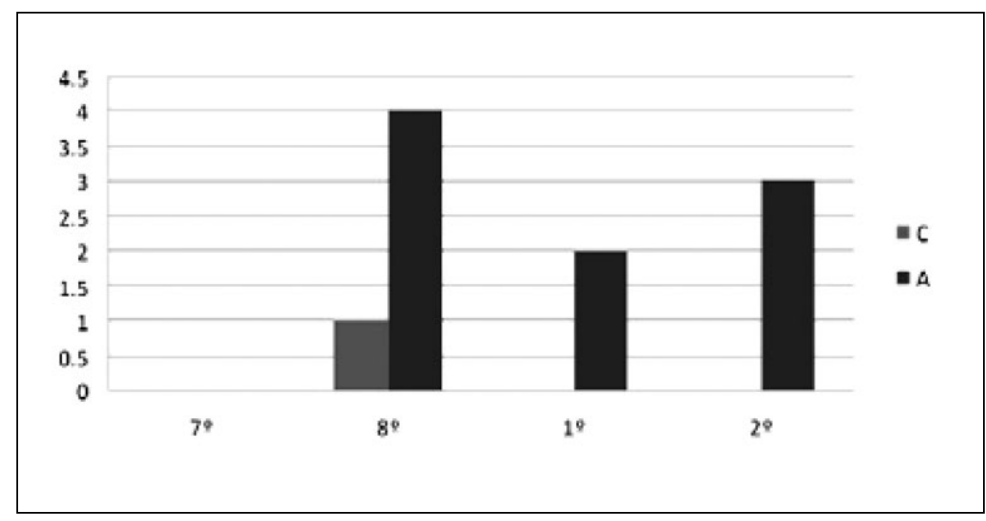

Fuente: Elaboración propia.

Solo en $8^{\circ}$ básico se presenta como contenido, estableciendo una definición y caracterización pasiva: "Promover una actitud (no beber alcohol, ser responsable, cuidar el entorno". Las actividades asociadas tienen que ver con lectura (y comprensión) del texto no lineal y producción de afiches sin fines de lucro. Si bien en términos numéricos no se aumenta 
gradualmente, existe presencia de actividades en $8^{\circ}$ básico (4), $1^{\circ}$ medio (2) y $2^{\circ}$ medio (3). En $1^{\circ}$ medio, se proponen 2 actividades:

- “Cómo se llama este tipo de texto?”, "En él, ¿cuál es la importancia de la imagen”, “¿Quién es el receptor del mensaje”.

- “¿Quiénes son los receptores del afiche? Justifica tu respuesta con elementos verbales o visuales”, “qué opinas de que -en el afiche- se apele a los hombres a terminar con la violencia en la pareja".

Las preguntas van orientadas hacia el reconocimiento de emisor y receptor de los mensajes, en desmedro de un análisis de los códigos que lo construyen. Es más bien, superficial. Sin embargo, se reconoce la progresión en los niveles como un aspecto coherente en el avance curricular.

La alfabetización mediática, como proceso, está al servicio del desarrollo de la competencia de lectura crítica de medios. Se presenta el resultado por cada texto escolar considerandos los niveles: funcional, analítico y crítico, obtenidos mediante la clasificación de contenidos y actividades propuestas.

\subsection{Niveles de alfabetización mediática de $7^{\circ}$ básico}

En este nivel se presenta la predominancia de actividades y contenidos que apuntan a un nivel funcional de medios, destacando la aproximación histórica y el consumo de medios, con 7 y 5 apariciones, respectivamente. Solo se presentan dos actividades de escritura: una de texto lineal; otra, no lineal. El nivel crítico se relega a 2 apariciones acerca de crítica de producción y 1 de autocrítica de la televisión chilena. En estos casos, se trata de actividades de lectura.

Figura 6: Niveles de alfabetización mediática ( $7^{\circ}$ básico).

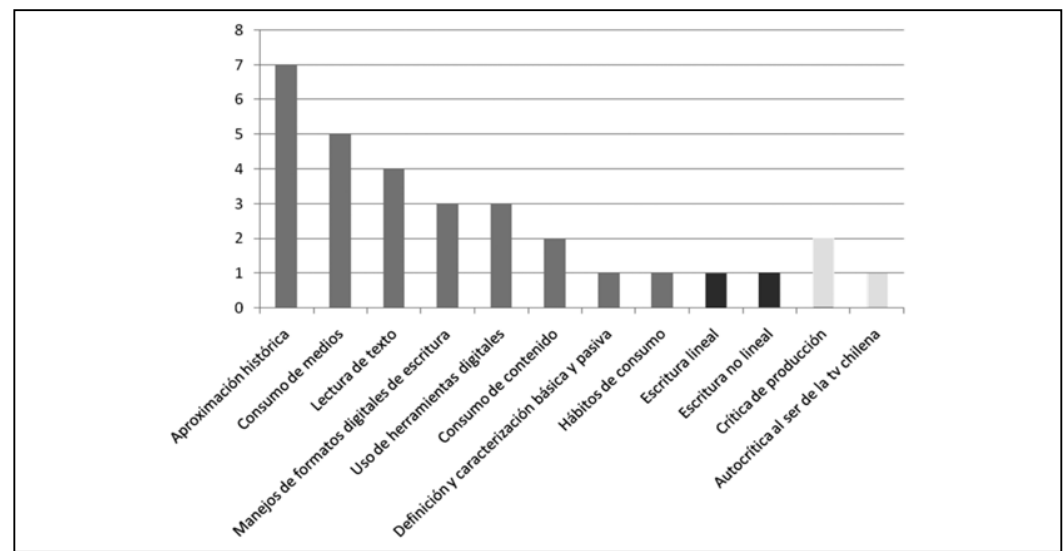

Fuente: Elaboración propia. 


\subsection{Niveles de alfabetización mediática de $8^{\circ}$ básico}

En este curso, el número de apariciones aumenta, presentándose la lectura de texto y el uso de herramientas digitales en 14 ocasiones. Al igual que en curso anterior, permanecen las actividades de escritura, las cuales aumentan a 7. Solo aparece una referencia al nivel crítico, en relación a la prevención del consumo y que consiste en el análisis de un afiche propagandístico que invita a la precaución en la entrega de información privada en las redes sociales.

Figura 7: Niveles de alfabetización mediática ( $8^{\circ}$ básico).

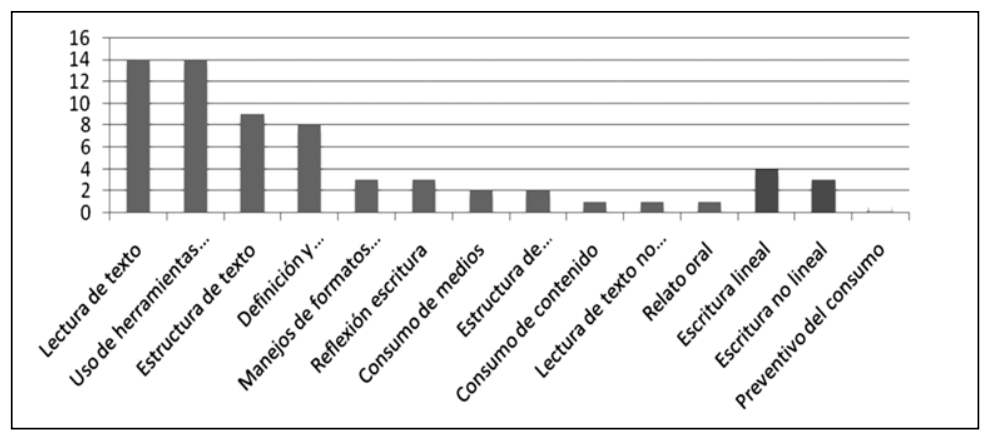

Fuente: Elaboración propia.

\subsection{Niveles de alfabetización mediática de $1^{\circ}$ medio}

En el nivel funcional, aumentan las actividades referidas a uso de herramientas digitales y lecturas de textos para mejorar comprensión lectora, con 17 y 15 apariciones respectivamente. También se incrementa el número de actividades referidas a escritura lineal (6) y no lineal (3). Asimismo, aumentan las instancias que corresponden al nivel crítico: crítica de publicidad, audiencia crítica y construcción de realidad; sin embargo, solo aparecen en una ocasión cada una.

Figura 8: Niveles de alfabetización mediática $\left(1^{\circ}\right.$ medio $)$.

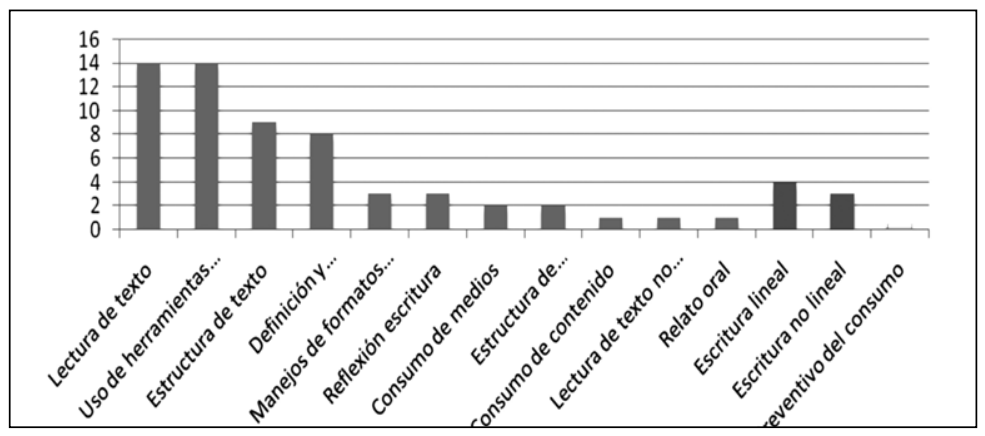

Fuente: Elaboración propia. 


\subsection{Niveles de alfabetización de $2^{\circ}$ medio}

En $2^{\circ}$ medio, aumenta el número de actividades y contenidos referidos al nivel funcional, destacando la lectura de texto y el uso de herramientas digitales, con 26 y 25 apariciones, respectivamente. En tanto, el nivel analítico incorpora (a diferencia de los cursos anteriores) la producción, como actividad audiovisual, por ejemplo, de programas de televisión y reportajes. Por último, la clasificación de contenidos y actividades ha ampliado su clasificación a crítica de poder, de producción y de publicidad. Además, aparece una alusión al consumo crítico y prevención del medio.

Figura 9: Niveles de alfabetización mediática $\left(2^{\circ}\right.$ medio $)$.

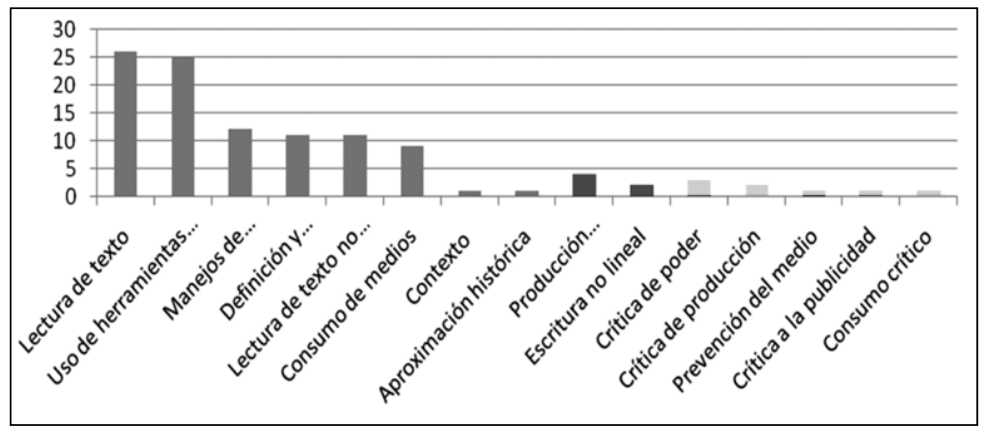

Fuente: Elaboración propia.

\section{Discusión y resultados}

Dentro de los contenidos encontrados emerge notoriamente el texto digital como contenido y actividad a realizar. Principalmente centrado en el manejo de formatos digitales de escritura y uso de herramientas digitales. Predomina, en estos casos, el uso de las herramientas de Microsoft Word, Microsoft Power Point, Internet y el Blogspot. Los estudiantes de hoy poseen un mejor manejo de las tecnologías, tienen mayor experticia de estas técnicas y conocen el uso de nuevos sistemas de comunicación. Por ende, el empleo de estos recursos favorece el trabajo docente y la comprensión de los contenidos por parte de los estudiantes. Sin embargo, el aumento de actividades no implica mayor complejidad ni criticidad por parte de los mismos.

En relación a la publicidad, se privilegia el consumo social y se omiten actividades orientadas al análisis de publicidad con fines comerciales. Si bien los términos de publicidad y propaganda se definen como excluyentes, el tratamiento que se hace de estos no considera las estrategias de persuasión, fundamentales para avanzar en una comprensión crítica.

Considerando los parámetros internacionales, la educación en medios debiese ser "con" y "sobre" los medios, más que "a través" de los medios. No obstante, la metodología propuesta para la enseñanza de lectura crítica de medios en textos escolares, según las 
actividades consignadas, apunta a una educación "a través" de los medios, predominando el nivel funcional instrumental de los medios en todos los textos escolares analizados. Con ello, Kendall y Mcdougall (2012: 22) señalan "existe una necesidad urgente de preparar los argumentos y focalizar la energía, ante el riesgo de que la alfabetización mediática se disperse en la bruma tecnológica".

El nivel analítico-descriptivo de los medios considera las actividades de diseño y producción de tipos de texto, principalmente de la entrevista. Además, de tipos de lineales como la crónica, no lineales (afiches), digitales (volante virtual), audiovisuales (documental y programa de televisión). Con ello, subyace la consideración no sólo de los textos lineales tradiciones, sino que existe apertura a la integración de formatos de distinta intencionalidad, pero que requieren de alfabetización mediática.

Por otra parte, escasamente se encuentran alusiones que se incluyan en el nivel críticocomprensivo (de las mediaciones): se trata especialmente de aquellas reflexiones que invitan a la prevención del medio y al consumo crítico. Es aquí donde es necesario integrar actividades, reflexión, lectura de textos con la finalidad de alcanzar lecturas críticas de los medios por parte de los estudiantes. Por ejemplo, que los estudiantes conozcan la producción de los mensajes mediáticos en distintos soportes tecnológicos y tradiciones pertenecientes a los medios de comunicación; poder e ideología en los mismos, entre otros.

Considerando lo anterior, con los contenidos y actividades propuestos por los textos escolares entregados por el Ministerio de Educación, se ve imposible un desarrollo de la competencia de lectura crítica de medios. Es necesario recordar que el texto escolar es un recurso de aprendizaje: no es la fuente de todo el conocimiento. He ahí el rol fundamental de cada docente, de su ejercicio profesional y su capacidad de planificar y organizar los contenidos y actividades.

\section{Fuentes consultadas}

Aguaded, J. (1997). "La televisión en el nuevo diseño curricular español”. Comunicar, vol. 8, pp. 97-109.

Bardin, L. (2002). Análisis de contenido. Madrid: Ediciones Akal.

Bonilla Loyo, E. (2006). Procesos de institucionalización y representaciones sociales del campo de la comunicación. Un estudio de caso [tesis doctoral en administración]. México D. F.: Universidad La Salle.

Buckingham, D. (2005). Educación en medios. Alfabetización, aprendizaje y cultura contemporánea. Barcelona: Paidós.

Cassany, D.

_(2006). Tras las líneas. Sobre la lectura contemporánea. Barcelona: Anagrama. 
_(2004). "Explorando las necesidades actuales de comprensión. Aproximaciones a la comprensión crítica”. Lectura y Vida. Vol. 25, núm. 2, pp. 6-23.

Del Valle, C. (2005). "Pedagogía de la comunicación. Los medios en las escuelas". Chasqui, vol. 89, pp. 32-39.

Del Valle, C. y Ojeda, X. (2013). "Educación económica y financiera para la formación inicial de profesores: herramientas conceptuales y didácticas”, pp. 43-54. En Sepúlveda Aravena, J. Educación económica y financiera para la formación inicial de profesores: herramientas conceptuales y didácticas. Extraída el 22/VII/2015 desde http://humanidades. ufro.cl/index.php/component/docman/doc_download/192-educacion-economica

Fainholc, B. (2005). "La lectura crítica en Internet". Lectura y Vida. Vol. 26, núm. 2, pp. $34-41$.

García Canclini, N. (1995). Consumidores y ciudadanos: conflictos multiculturales de la globalización. México D. F.: Grijalbo.

Gilster, P. (1997). Digital literacy. Nueva York: Wiley.

Giménez, G. (1981). Poder, Estado y discurso. Perspectivas sociológicas y semiológicas del discurso político-jurídico. México D. F.: Universidad Nacional Autónoma de México.

Kendall,A. y McDougall, J.(2012). “Alfabetización mediática crítica en la postmodernidad”. Comunicar. Vol. XIX, núm. 38, pp. 21-29.

Kellner, D. y Share, J. (2007). "Critical media literacy, Democracy, and the Reconstruction of Education", pp. 3-23. En Macedo, D. y Steinberg, S. (eds.). Media Literacy: A Reader. Nueva York: Peter Lang Publishing.

Livingstone, $\mathrm{S}$.

_(2008). "Engaging with Media - A Matter of Literacy?”. Communication, Culture and Critique. Vol. 1, núm. 1, pp. 51-62.

_(2004). "What is Media Literacy?”. Intermedia. Vol. 32, núm. 3, pp. 18-20.

Livingstone, S. y Bovill, M. (1999). Young People, New Media: report of the research project Children Young People and the Changing Media Environment. Research report, Department of Media and Communications, London School of Economics and Political Science, London UK.

Luke, A. y Freebody, P. (1997). "Further Notes on Four Resources Model”. Extraída el 22/ VII/2015 desde http://www.readingonline.org/research/lukefreebody.html

MINEDUC. (2009). Objetivos fundamentales y contenidos mínimos obligatorios de la educación básica y media. Santiago de Chile: MINEDUC. 
Murphy, D. (2010). "Euromeduc: the Second Congress of Media Education Practitioners". Media Education Journal, vol. 47, pp. 23-27.

Pérez Tornero, J. (1994). El desafio educativo de la televisión. Barcelona: Paidós.

Pineda, A. (2012). "Propaganda y educación. Criterios de diferenciación conceptual". Análisis, vol. 39, pp. 131-148.

Pozo, M.; Gutiérrez, J. y Rodríguez, C. (2007). "El uso del método Delphi en la definición del los criterios para una formación de calidad en animación sociocultural y tiempo libre". Revista de investigación educativa, vol. 2, pp. 351-366.

Pujolà, J. y Montmany, B. (2010). "Más allá de lo escrito: la hipertextualidad y la multimodalidad en los blogs como estrategias discursivas de la comunicación digital. Alfabetización mediática y culturas digitales". Conferencia llevada a cabo en el I Congreso Euro Iberoamericano de Alfabetización Mediática y Culturas Digitales. Sevilla, España.

Sánchez, J. y Aguaded, J. (2009). "Educación mediática y espectadores activos: estrategias para la formación”. Análisis, núm. 39, pp. 131-148.

Serrano de Moreno, S. y Madrid de Forero, A. (2008). "Competencias de lectura crítica. Una propuesta para la reflexión y la práctica”. Acción Pedagógica. Vol. 16, núm. 1, pp. 58-68.

Vidal, M. y González, M. (2011). Lenguaje y Comunicación. Texto del estudiante. $1^{\circ}$ Educación Media. Santiago de Chile: Santillana del Pacífico S. A.

Watson, G. y Aguilera, N. (2012). Lenguaje y Comunicación. Texto del estudiante. $8^{\circ}$ Educación. Santiago de Chile: Santillana del Pacífico S. A.

Zarandona, E. y otros (2008). "La alfabetización audiovisual entre adolescentes vascos: implicaciones para las prácticas educativas y propuesta de intervención". Nueva Época, vol. 9, pp. 119-143. 